\title{
Aquaporin based biomimetic membrane in FO: chemical cleaning resistance and practical operation
}

Zhenyu $\mathrm{Li}^{1, *}$, Rodrigo Valladares Linares ${ }^{2, *}$, , Szilard Bucs ${ }^{2}$, Luca Fortunato ${ }^{2}$, Claus Hélix-Nielsen ${ }^{3,4,5}$, J.S. Vrouwenvelder $^{2,6,7}$, NorEddine Ghaffour ${ }^{2}$, TorOve Leiknes ${ }^{2}$, Gary Amy ${ }^{8,9}$

${ }^{1}$ College of Food Science and Engineering, Northwest A\&F University, Yangling, Shaanxi 712100, China

${ }^{2}$ Water Desalination and Reuse Center, Division of Biological and Environmental Science and Engineering, King Abdullah University of Science and Technology, Thuwal 23955-6900, Saudi Arabia

${ }^{3}$ Department of Environmental Engineering, Technical University of Denmark, Bygningstorvet 115, DK2800 Kgs. Lyngby, Denmark

${ }^{4}$ Laboratory for Water Biophysics and Membrane Technology, Faculty of Chemistry and Chemical Engineering, University of Maribor, Maribor, Slovenia

${ }^{5}$ Aquaporin A/S, Ole Maaløes Vej 3, DK2200 Copenhagen, Denmark

${ }^{6}$ Delft University of Technology, Faculty of Applied Sciences, Department of Biotechnology, Delft, The Netherlands

${ }^{7}$ Wetsus, Centre of Excellence for Sustainable Water Technology, Leeuwarden, The Netherlands

${ }^{8}$ College of Engineering and Science, Clemson University, Clemson, SC 29634, USA

${ }^{9}$ Chemical and Biomolecular Engineering, National University of Singapore, 119077, Singapore

*Corresponding author's email: lizhenyu@nwafu.edu.cn; rvalladares@rotoplas.com

\begin{abstract}
Aquaporin plays a promising role to prepare a high performance biomimetic forward osmosis membrane. However, aquaporin as a protein also has a risk of denaturation caused by various chemicals, resulting in a possible decay of membrane performance. The present study tested a novel aquaporin biomimetic membrane in simulated membrane cleaning processes. The effects of cleaning detergents on water flux and salt rejection were evaluated. The membrane showed a good resistance to chemical detergents tested. The water flux after chemical cleaning showed significant increases, particularly after cleaning with $\mathrm{NaOCl}$ and Alconox. Changes in the membrane structure and increased hydrophilicity in the surrounding areas of the aquaporin protein channel may be accountable for the increase in water permeability. The membrane shows stable salt rejection up to $99 \%$ after all cleaning solutions were used. A 15-day experiment with secondary wastewater effluent as the feed and seawater as the draw solution showed a stable flux and high salt rejection. The average total organic carbon rejection from wastewater after 15-day test was $90 \%$. The results demonstrated that the aquaporin based biomimetic forward osmosis membrane exhibits chemical resistance for most common detergents used in membrane cleaning procedures, maintaining a stable flux and high salt rejection.
\end{abstract}


Keywords: biomimetic membrane; aquaporin; desalination; forward osmosis; chemical stability.

\section{Introduction}

With the scarcity of clean water in many regions of the world, seawater and brackish water desalination will play a crucial role for future water supplies. The current leading membrane desalination technology by reverse osmosis (RO) is an energy intensive process. Forward osmosis (FO) is a novel membrane-based process that can potentially reduce energy consumption to conventional water treatment and desalination. In FO process, water is extracted from a lower concentration feed solution (FS) into a higher concentration draw solution (DS), driving by the osmotic pressure difference, consequently results in the concentration of the FS and dilution of the DS.

Aquaporin is a channel-forming transmembrane glycoprotein with the ability to mediate water entry or release through cell membrane, driving by a transmembrane osmotic gradient. Aquaporin can thus be defined as a water channel protein. This property of facilitating a high water permeability while maintaining an excellent solute selectivity is a feature that cannot be found in any artificial polymeric membrane and has served as inspiration for designing biomimetic membranes [1,2].

Aquaporin protein has been recently embedded into the active layer of thin-film composite (TFC) membranes [3]. Aquaporin serves as pore-forming protein that facilitates water diffusion. With the passive facilitated transport, the protein has a stable geometrical structure during operation and can achieves single channel turnover rates up to $10^{9}$ water molecules per second [4]. Therefore, aquaporin-assisted membranes potentially have very high permeabilities compared to conventional membranes [5]. Aquaporin based biomimetic membranes have been shown to provide a potential benefit for water purification and desalination [6]. However, as a protein, aquaporin can be affected by surrounding conditions such as temperature, $\mathrm{pH}$ and presence of chemicals that may impact its water transport properties. It is becoming important to examine how an aquaporin membrane functions under 'realistic' conditions.

A literature review shows that few studies have been conducted in terms of practical applications of aquaporin based membranes. The rejection of small neutral organic compounds in water has been tested using aquaporin based biomimetic membranes [7]. The performance of this membrane was compared with a cellulose triacetate (CTA) FO membrane, showing that the biomimetic membranes have rejection values higher than those from the CTA membrane. The application of aquaporin based biomimetic membranes has shown promising results in the various applications including FO processes $[6,8]$. Nevertheless, the stability of membranes during practical conditions (i.e. the use of real wastewater and seawater in the FO process) and after chemical cleaning procedures has not been addressed. 
In the present study, a novel aquaporin based biomimetic membrane was tested with different detergents to simulate membrane chemical cleaning and aging. The stability of the membrane was evaluated by water flux measured before and after simulated membrane cleaning processes. A long-term experiment lasting 15 days was also conducted with secondary wastewater effluent as the FS and Red Sea water as the DS to test the membrane under realistic conditions.

\section{Materials and Methods}

\subsection{Forward osmosis (FO) membranes}

The aquaporin based biomimetic membrane was provided by Aquaporin A/S (Copenhagen, Denmark). It is a (TFC membrane with incorporated aquaporin channels immobilized in vesicles. The vesicles are stabilized in the polyamide (PA) thin film (i.e. active layer) on the surface of a porous polysulfone support layer [3]. Figure 1 shows SEM images of the top surface, bottom surface and a cross-section of the aquaporin based biomimetic membrane. More details on the membrane fabrication procedure can be found elsewhere [3].

An equivalent TFC membrane without aquaporin proteins was provided by Woongjin Chemicals (Korea). Details on the structure and characteristics of the membrane can be found in literature [9].

\section{Figure 1}

\subsection{FO experimental setup}

A bench-scale FO system was used in this study. The system consisted of a cross-flow FO cell using a $2 \mathrm{~cm} \times 10 \mathrm{~cm}$ flat-sheet membrane. Experiments were operated at a flow rate of 0.2 $\mathrm{L} \cdot \mathrm{min}^{-1}$,(i.e. a cross-flow velocity of $\left.0.084 \mathrm{~m} \cdot \mathrm{s}^{-1}\right)$. 1 liter of the FS and 1 liter of the DS were used for each test. The details of system configuration has been described in previous FO studies $[10,11]$. All experiments were performed at a temperature of $21 \pm 1{ }^{\circ} \mathrm{C}$. The conductivity of the FS and DS was monitored by a conductivity meter (WTW, Germany). All water permeation tests were performed under a pH range between 5.3 and 6.5. The FS was deionized (DI) water and the DS was a $4 \% \mathrm{NaCl}$ solution that simulates Red Sea water salt concentration.

The effect of membrane chemical cleaning in the FO process was tested by using analog chemical detergents. All chemicals used were purchased from Sigma Aldrich (Germany). Five chemical cleaning solutions were used independently in this study: acid cleaning with $0.1 \%$ hydrochloric acid $(\mathrm{HCl})$ at a $\mathrm{pH}$ of 1.4 , biocide cleaning with $0.5 \%$ sodium hypochlorite $(\mathrm{NaOCl})$ at a pH of 11.6, chelating cleaning of $5 \mathrm{mM}$ disodium ethylenediaminetetraacetic acid (EDTA) at 
a $\mathrm{pH}$ of 8.2 , anionic surfactant cleaning consisting of $2 \mathrm{mM}$ sodium dodecyl sulfate (SDS) at a pH of 9.1, and a commercial anionic detergent solution (Alconox) of $1 \%$ at a pH of 9.3, composed of sodium dodecylbenzenesulfonate (10-30\%) commonly known as linear alkylbenzene sulfonate (LAS), sodium carbonate (7-13\%), tetrasodium pyrophosphate (10-30\%) and sodium phosphate (10-30\%). These cleaning methods were chosen as they are the most common techniques used to clean membranes in seawater desalination and wastewater recovery processes [12-16].

For the long-term experiment, the FS was secondary wastewater effluent collected from the AlRuwais wastewater treatment plant in the city of Jeddah, Saudi Arabia, with a total organic carbon (TOC) of $4.95 \pm 0.20 \mathrm{mg} \cdot \mathrm{L}^{-1}$, conductivity of $3.3 \mathrm{mS} \cdot \mathrm{cm}^{-1}$ and a $\mathrm{pH}$ of 7.5 . The DS was raw seawater from the Red Sea with a TOC of $0.98 \pm 0.07 \mathrm{mg} \cdot \mathrm{L}^{-1}$, conductivity of $59.7 \mathrm{mS} \cdot \mathrm{cm}^{-1}$ and a $\mathrm{pH}$ of 7.8 .

\subsection{Testing protocol of chemical cleaning and evaluation of membrane performance}

The testing protocol of chemical cleaning assessment is described in Figure 2 . The baseline flux and salt rejection were determined for each membrane coupon used in the experiments. These experiments used DI water as the $\mathrm{FS}$ and $4 \% \mathrm{NaCl}$ as the DS. Afterwards, chemical cleaning was performed for $0.5 \mathrm{~h}$ at the FS side of the membrane; DI was recirculated at the DS side. Immediately after cleaning, the membrane was flushed with DI water to remove the remaining chemical agent. Finally, the average flux and salt rejection were calculated for a period of $2 \mathrm{~h}$ to study the effect of each cleaning solution on the membrane performance. Results presented in this study show the average value of three independent tests for each condition.

\section{Figure 2}

As membrane orientation plays a crucial role impacting the propensity of fouling during FO process, each cleaning experiment was conducted with two membrane orientations: AL-FS (the membrane active layer facing the FS) and AL-DS (the active layer facing the DS).

A long-term running for 15 days was also conducted with secondary wastewater effluent as the FS and Red Sea water as the DS under a semi-batch mode. Each batch was operated for 24 hours. Then concentrated FS and diluted DS were replaced by fresh aliquots and operation continued to the next batch. The long-term process was operated under AL-FS mode which has been proven to be the most effective in preventing fouling of an FO membrane $[13,17,18]$. Total organic carbon (TOC) was measured by an Organic Carbon Analyzer (TOC-VCPH, Shimadzu, Japan).

The salt rejection from the DS to the FS side was estimated by the conductivity measured in the DS and FS reservoir, which was converted to salt concentration. Equation 1 was used to calculate salt rejection considering that the dilution of the DS in each experiment is negligible: 


$$
\text { Salt Rejection }(\%)=\left(1-\frac{C_{\mathrm{FS}}}{C_{D S}}\right) \times 100
$$

where $C_{F S}$ is the calculated salt concentration in the FS reservoir after each experiment, and $C_{D S}$ is the calculated salt concentration in the DS reservoir at the beginning of each experiment. The initial conductivity of the FS reservoir is lower than $1.0 \mu \mathrm{S} \cdot \mathrm{cm}^{-1}$ which is negligible.

Equation 2 was used to calculate the salt rejection for the long-term experiment, where a secondary wastewater was used as FS and seawater as DS. The salt rejection was determined by the conductivity in the DS reservoir, and relating it to the loss of salt in each batch to the feed side:

$$
\text { Salt Rejection }(\%)=1-\left(1-\frac{C_{\mathrm{DS} 2} \times V_{D S 2}}{C_{\mathrm{DS} 1} \times V_{D S 1}}\right) \times 100
$$

where $C_{D S 1}$ and $C_{D S 2}$ are the salt concentration of DS before and after each cycle respectively, and $V_{D S 1}$ and $V_{D S 2}$ are the bulk volumes of DS before and after each cycle, respectively.

\subsection{Characterization of membrane surface}

The surfaces of the membrane before and after chemical cleaning were characterized by Fourier Transform Infrared Spectroscopy in the attenuated total reflectance mode (ATR-FTIR, Perkin Elmer Spectrum 100). An electrokinetic analyser for solid surface analysis (Anton Paar, Austria) was used to determine the zeta potential of the membrane surface using $10 \mathrm{mM} \mathrm{NaCl}$ solution as electrolyte. The results were calculated by the Helmholtz-Smoluchowski equation [19].

\section{Results and Discussion}

Experiments were conducted to determine the stability of an aquaporin based biomimetic membrane, evaluating salt rejection and water flux before and after various chemical cleanings. Surface characterization was performed afterwards to study the effects of each chemical on the composition and charge of the membrane active layer. Each chemical cleaning solution showed to have a different effect on the performance of the biomimetic membrane.

\subsection{Salt rejection and water flux}

The normalized water flux for the baseline experiment is shown as 1.0 in Figure 3 for both AL-FS and AL-DS membrane orientations. Similarly, the normalized water flux after each test of chemical cleaning is also shown for both membrane orientations. It can be seen that for all the cleaning agents the flux was either similar or higher to the baseline flux. The $\mathrm{HCl}$ cleaning increased the flux by $9.8 \%$ in AL-FS mode and $20 \%$ in AL-DS mode. Cleaning with $\mathrm{NaOCl}$ showed the highest water flux increase among all cleaning agents, with a $66 \%$ and $100 \%$ increase compared to the baseline value. A slight improvement was found with sodium EDTA solution, 
achieving an increase in flux of $1.5 \%$ and $5.1 \%$ for AL-FS and AL-DS mode, respectively. After the SDS cleaning, the water flux increased $28 \%$ and $6.5 \%$ for each membrane orientation. After cleaning the membrane with Alconox detergent, the water flux showed an increase of $75 \%$ in AL-FS mode and $31 \%$ in AL-DS mode.

\section{Figure 3}

The rejection to the back diffused $\mathrm{NaCl}$ from the DS to $\mathrm{FS}$ was above $98 \%$ for all the tests done as baseline (before chemical cleaning) as shown in Figure 4a (AL-FS mode) and Figure 4b (AL-DS mode). Salt rejection was not affected by any chemical cleaning as described by the values of rejection measured after chemical cleaning, being the largest difference lower than $0.5 \%$. These tests reveal the performance stability of aquaporin based biomimetic membranes to reject $\mathrm{NaCl}$ even after chemical cleaning.

\section{Figure 4}

\subsection{Mechanisms related to membrane performance change}

$\mathrm{HCl}$ and $\mathrm{NaOCl}$ cleaning had a higher impact on the membrane support side. Compared with the flux increase when the active layer was chemically cleaned, an increase of $10 \%$ in the water flux was achieved after the support layer was cleaned by $\mathrm{HCl}$ solution. In the case of $\mathrm{NaOCl}$, the increase was $35 \%$. These results indicate that the impact on the membrane structure might be more significant than the impact on the embedded aquaporin proteins in the AL surface when $\mathrm{HCl}$ and $\mathrm{NaOCl}$ were used as cleaning detergents.

Generally, TFC membranes have good chemical stability and minimum degradation towards $\mathrm{pH}$, hydrolysis and biodegradation [20-22]. HCl can induce a physical change of polysulfone support and open the pore of support layer while the chemical degradation of $\mathrm{PA}$ active layer by $\mathrm{HCl}$ is negligible [23]. The change of porosity in support layer can reduce internal concentration polarization (ICP) during FO process, consequently increased flux. During $\mathrm{NaOCl}$ chemical cleaning, $\mathrm{N}-\mathrm{H}$ hydrogen of membrane material is replaced by chlorine while the hydrogen bonds between $\mathrm{C}=\mathrm{O}$ and $\mathrm{N}-\mathrm{H}$ are weakened or even destroyed. The rotational freedom and flexibility of the crosslinked polymer chains are increased [24, 25]. Meanwhile, chlorination promotes the amide $\mathrm{C}-\mathrm{N}$ bond hydrolysis and makes the membrane more hydrophilic [24-26].. As a consequence of these structural changes and enhanced hydrophilicity of the membrane after chemical cleaning by $\mathrm{NaOCl}$ solution, the restrictions of water passage through the membrane is decreased, resulting in an improved water permeability [27]. In addition, $\mathrm{NaOCl}$ can cause $\mathrm{C}-\mathrm{S}$ bond scission in polysulfone molecules, thus results in larger pore size in the polysulfone porous support, which can improve flux of FO process by reducing ICP $[28,29]$. 
Furthermore, the size of nodules in the PA layer of the membrane decreases and the internodular spaces is filled by the arisen polymer chains from adjacent nodules because of the interaction between chemical cleaning agents and polymer chains in the PA layer of TFC membrane [30]. The PA composite membrane may have been transformed into more homogeneous membrane, explaining the changes in water permeability during FO filtration.

Related to the embedded aquaporin modification, it has been reported that aquaporin proteins show a variation in water permeability in relation to the $\mathrm{pH}$ of the solution, depending on the type of structure of the protein [31]. During $\mathrm{NaOCl}$ (oxidizing and basic $\mathrm{pH}$ ) and acid (low $\mathrm{pH}$ ) cleaning, the position of histidines which is a $\alpha$-amino acids with an imidazole functional group in the aquaporin protein might be responsible of the changes in water permeability. Histidines can "tune" the pH sensitivity of different aquaporin proteins toward acid or alkaline ranges [32].

For the cleaning solutions containing SDS and Alconox, the impact was higher on the membrane active layer. When the active layer of membrane was cleaned by SDS solution, it showed an $21 \%$ increase of water flux compared to cleaning the support layer. The increase of flux was $44 \%$ when comparing both membrane orientations with the Alconox cleaning. These results suggest that these cleaning agents have an effect on the active layer and possibly on the aquaporin proteins embedded on it. SDS can remove the unreacted water-soluble monomers, thus increases the free volume size and fractional free volume in the membrane active layer and improve the water flux [33].

A test was made to compare the effect of Alconox cleaning solution on an unmodified TFC FO membrane and an aquaporin based biomimetic membrane. Results are shown in Figure 5. Cleaning an unmodified TFC membrane with Alconox has almost no effect on the water flux. When the membrane modified with aquaporin proteins is cleaned, the water flux increases $75 \%$ compared to the baseline test, which could suggest that the proteins are being affected by the cleaning agent. It should be noted that Alconox detergent can cause the enlargement of pore size in polysulfone support of a TFC FO membrane after a long-term (144 hours) exposure which resulted in an increase of FO flux [34]. The negligible effect on the water flux of unmodified TFC FO membrane in the present study could be attributed to the short exposure time.

Alconox is a detergent composed mainly of phosphates and LAS. The effect of sodium phosphate on the aquaporin based biomimetic membrane was tested as well and compared to the results with Alconox cleaning (Figure 5). The water flux after cleaning with sodium phosphate did not change significantly compared to the baseline, showing that phosphates have almost no effect on the aquaporin protein embedded on the membrane surface. Still surfactants present in the Alconox detergent (i.e. LAS) could be responsible for the aquaporin protein modification, resulting in a change on the water flux of the membrane.

\section{Figure 5}


Studies for the conformation of protein in SDS complex showed that the molecules of SDS have marginal effect on the conformation of aquaporin including the structure of water channel. However, Borgnia et al. [35] reported that longer incubation time (>24 hours) in $1 \%$ SDScontaining sample buffer caused the dissociation of the tetramer forming the aquaporin protein in E. coli even without reducing agents. Even though SDS is a denaturing anionic detergent, it is not able to change the aquaporin secondary structure [36]. Also Hansen et al. [37] found that SDS solution can induce the reconstitution and helical rearrangements of aquaporins into large lipid vesicles, but the secondary structure of the protein is not affected and the aquaporin vesicles can work as a promising procedure to stabilize the protein in membrane surfaces.

In principle aquaporins could lose water permeability when single SDS molecules or structures attach to the hydrophilic ends of the water pore, surrounding areas or even inside the channels, consequently block the water channel of aquaporins [36]. Single SDS molecules were present under our experimental conditions as SDS is not forming micelles at $2 \mathrm{mM}$ concentration since the critical micelle concentration of SDS is $8.2 \mathrm{mM}$. However, decrease in water flux before and after cleaning was not observed (see Figure 3), thus indicating that this effect is negligible.

LAS in the Alconox detergent forms micelles at lower concentrations than SDS. The average concentration of LAS in the solution was $2.5 \mathrm{mM}$, and the critical micelle concentration of LAS is around $1.1 \mathrm{mM}$ [38]. Due to this effect, chemical cleaning with Alconox showed a higher positive impact on water flux compared to SDS cleaning.

The reduction of membrane surface tension caused by detergents may also be responsible for the higher water flux obtained after cleaning. Along with the reduced surface tension, detergents increase the hydrophilicity of neighboring areas of aquaporin structures, thereby possibly increasing the local water permeabilities. This result may not be observed in the TFC membrane without aquaporin modification because the whole membrane system is different when the vesicles are present.

Although the dissociation of aquaporin protein may influence the water transport across the modified membrane, our results suggest that there is no denaturation process undergoing since the protein functionality was not significantly affected. The water flux increased and salt rejection is stable. The higher flux observed after cleaning may be accounted for a surface tension reduction.

\subsection{Membrane surface characterization after chemical cleaning}

To determine if the membrane surface underwent modification due to the chemical cleaning, its composition and charge were analyzed by FTIR and zeta potential measurements. No structural changes on the surface of the aquaporin based biomimetic membrane could be observed on SEM imaging after each chemical cleaning experiment (data not shown). 
Figure 6 shows ATR-FTIR spectrum of the active layer of a new aquaporin modified membrane and following each chemical cleaning procedure. No significant change on the chemical structure (i.e. functional groups) was observed, confirming that the biomimetic membrane was chemically stable after the cleaning procedures. Generally, the amide groups on the TFC membrane is resistant to the hydrolysis [39]..

\section{Figure 6}

The zeta potentials of a virgin aquaporin based biomimetic membrane as the baseline and the chemically cleaned samples were calculated by streaming potential. Results are shown in Figure 7. At the $\mathrm{pH}$ range from 5.3 to 6.5 , zeta potential is considerably more negatively charged when the membrane was cleaned with acid $(\mathrm{HCl})$ and $\mathrm{NaOCl}$. Increase of water flux after chemical cleaning can be explained by the changes of streaming potential and enhanced hydrophilicity of membranes after hypochlorite and acid cleaning [40, 41].

The membrane surface charge plays a significant role in membrane performance. The negatively charged surface of aquaporin based biomimetic membrane provides an electrostatic repulsive force on anions. Cations are also rejected in order to maintain the electroneutrality. The trend of zeta potential can illustrate the tendency of solute rejection by charge repulsion and explain the variation of flux and rejection [42]. Since the negative charge of membranes is enhanced after chemical cleaning with acid and $\mathrm{NaOCl}$, electrostatic repulsion increases between $\mathrm{Na}^{+}$and the membrane with a more hydrophilic surface. Particularly, the increased negative charge caused by $\mathrm{N}$-chlorination consumption of terminal amino groups after chlorination in hypochlorite treatment can contribute to the improvement of flux and salt rejection $[43,44]$.

For the cleaning experiments using EDTA, Alconox and SDS, a slight decrease in the charge can be observed at the $\mathrm{pH}$ range used. In the case of the anionic surfactants (Alconox and SDS), the decrease is an expected effect due to the binding of molecules on the surface of membrane with the negative charged hydrophilic tail exposed to the water. Salt rejection, thus, remains very high (>98\%) after chemical cleaning.

Figure 7

\subsection{Seawater desalination and wastewater recovery by forward osmosis process}

During a 15-day FO process with secondary wastewater effluent as the FS and seawater as the DS, both water flux and salt rejection were observed to be stable. The average water flux was 4.6 $\mathrm{L} \cdot \mathrm{m}^{-2} \cdot \mathrm{h}^{-1}$ and the average salt rejection $98.5 \%$ (Figure 8). However, both water flux and salt 
rejection in this test was lower than those observed for the conditions used in the cleaning solutions tests. The reduction of water flux was due to the formation of a fouling layer on the membrane surface at the FS side when secondary wastewater effluent was used as the FS. The lower flux consequently resulted in an increased back diffusion of salt from the DS [45]. Therefore, a decrease in salt rejection is also observed. The average TOC rejection from wastewater in 15 days was $90.7 \%$.

\section{Figure 8}

CTA FO membranes have been a benchmark for several years. Compared to previous experiments using a CTA membrane where a secondary wastewater effluent was the FS and seawater was the DS, the water flux values using an aquaporin based biomimetic membrane was found to be higher and more stable $[13,46]$. Flux was $3.4 \mathrm{~L} \cdot \mathrm{m}^{-2} \cdot \mathrm{h}^{-1}$ for the CTA membrane and $4.6 \mathrm{~L} \cdot \mathrm{m}^{-2} \cdot \mathrm{h}^{-1}$ in this study under similar testing condition. In terms of salt rejection, the aquaporin based biomimetic membrane also showed a higher rejection than CTA membranes, due to the ability of aquaporin proteins to selectively reject salt ions and allow water passage through the aquaporin water channel.

\subsection{Implications}

Results from this study indicate that aquaporin based biomimetic membranes appear to have a superior performance in FO processes for seawater desalination and wastewater recovery compared to conventional CTA FO membranes. However, more tests using real conditions are required for a full assessment. The results found in this study suggest that an increase in water flux of biomimetic membranes may be feasible by appropriate chemically treated prior to its use. For example, a quick flush with a cleaning agent such as SDS or LAS may improve the performance of aquaporin proteins to transport water, without compromising salt rejection. Results from the cleaning tests suggest that cleaning steps required, as per system operation, will not adversely affect an aquaporin based biomimetic membrane, where a selective water flux with high salt rejection are expected to be maintained. Verification is required in cleaning tests conducted under operation with real solutions (i.e. real wastewater and seawater).

\section{Conclusions}

An aquaporin based biomimetic membrane was tested by using different cleaning solutions to simulate membrane chemical cleaning processes. The stability of the membranes was evaluated. The membrane showed a good chemical stability when tested with different detergents and a wide $\mathrm{pH}$ range (1.4 to 11.7). EDTA showed a negligible effect on the water flux of the membrane. $\mathrm{HCl}, \mathrm{NaOCl}, \mathrm{SDS}$ and Alconox cleaning showed to increase the water flux of aquaporin based biomimetic membranes. The variation of flux was mainly attributed to the change of TFC membrane property when $\mathrm{HCl}, \mathrm{NaOCl}$ and SDS were used as the detergents while Alconox showed more impact on aquaporin. Very high salt rejection (>98\%) even after 
chemical cleaning were observed for all tested cleaning solutions. However, the significant change of flux caused by the detergents would be a potential challenge for the membrane aging in the future long-term implementation.

Initial tests with secondary wastewater effluent as the FS and natural seawater as the DS demonstrated a stable operation (i.e. water flux and salt rejection) and higher performance compared to a CTA membrane under similar testing conditions. Fouling layer formed at the FS side can cause a minor water flux reduction. More tests are necessary to validate these results for long-term operation and possible effects. Cleaning protocols may have on the process with real fouling conditions.

\section{Acknowledgements}

The present research was supported by King Abdullah University of Science and Technology in Saudi Arabia and Northwest A\&F University in China. We thank Aquaporin A/S for the valuable discussions and providing us with the aquaporin based biomimetic membranes.

\section{References:}

[1] P. Agre, G.M. Preston, B.L. Smith, J.S. Jung, S. Raina, C. Moon, W.B. Guggino, S. Nielsen, Aquaporin CHIP: The archetypal molecular water channel, AM.J.PHYSIOL. 265 (1993) 463476.

[2] F. Wicaksana, A.G. Fane, C. Tang, R. Wang, Biomimetic Membranes for Sensor and Separation Applications, (2012).

[3] Y. Zhao, C. Qiu, X. Li, A. Vararattanavech, W. Shen, J. Torres, C. Hélix-Nielsen, R. Wang, X. Hu, A.G. Fane, C.Y. Tang, Synthesis of robust and high-performance aquaporin-based biomimetic membranes by interfacial polymerization-membrane preparation and RO performance characterization, J. Memb. Sci. 423-424 (2012) 422-428.

[4] M. $\varnothing$. Jensen, O.G. Mouritsen, Single-channel water permeabilities of Escherichia coli aquaporins AqpZ and GlpF., Biophys. J. 90 (2006) 2270-2284.

[5] M. Kumar, M. Grzelakowski, J. Zilles, M. Clark, W. Meier, Highly permeable polymeric membranes based on the incorporation of the functional water channel protein Aquaporin Z., Proc. Natl. Acad. Sci. U. S. A. 104 (2007) 20719-20724.

[6] C.Y. Tang, Y. Zhao, R. Wang, C. Hélix-Nielsen, A.G. Fane, Desalination by biomimetic aquaporin membranes: Review of status and prospects, Desalination. 308 (2013) 34-40.

[7] H.T. Madsen, N. Bajraktari, C. Hélix-Nielsen, B. Van der Bruggen, E.G. Søgaard, Use of biomimetic forward osmosis membrane for trace organics removal, J. Memb. Sci. 476 (2015) 469-474. 
[8] C. Tang, Z. Wang, I. Petrinić, A.G. Fane, C. Hélix-Nielsen, Biomimetic aquaporin membranes coming of age, Desalination. 368 (2015) 89-105.

[9] W. Fam, S. Phuntsho, J.H. Lee, H.K. Shon, Performance comparison of thin-film composite forward osmosis membranes, Desalin. Water Treat. 51 (2013) 6274-6280.

[10] Z.Y. Li, V. Yangali-Quintanilla, R. Valladares-Linares, Q. Li, T. Zhan, G. Amy, Flux patterns and membrane fouling propensity during desalination of seawater by forward osmosis, Water Res. 46 (2012) 195-204.

[11] R. Valladares Linares, Z.Y. Li, S. Sarp, Y.G.G. Park, G. Amy, J.S. Vrouwenvelder, Higher boron rejection with a new TFC forward osmosis membrane, Desalin. Water Treat. (2014) $1-7$.

[12] R. Valladares Linares, Z.Y. Li, V. Yangali-Quintanilla, Q. Li, G. Amy, Cleaning protocol for a FO membrane fouled in wastewater reuse, Desalin. Water Treat. 51 (2013) 4821-4824.

[13] R. Valladares Linares, V. Yangali-Quintanilla, Z. Li, G. Amy, NOM and TEP fouling of a forward osmosis (FO) membrane: Foulant identification and cleaning, J. Memb. Sci. 421422 (2012) 217-224.

[14] S. West, H. Horn, W. a M. Hijnen, C. Castillo, M. Wagner, Confocal laser scanning microscopy as a tool to validate the efficiency of membrane cleaning procedures to remove biofilms, Sep. Purif. Technol. 122 (2014) 402-411.

[15] K.J. Varin, N.H. Lin, Y. Cohen, Biofouling and cleaning effectiveness of surface nanostructured reverse osmosis membranes, J. Memb. Sci. 446 (2013) 472-481.

[16] B.P. Espinasse, S.R. Chae, C. Marconnet, C. Coulombel, C. Mizutani, M. Djafer, et al., Comparison of chemical cleaning reagents and characterization of foulants of nanofiltration membranes used in surface water treatment, Desalination. 296 (2012) 16.

[17] B. Mi, M. Elimelech, Chemical and physical aspects of organic fouling of forward osmosis membranes, J. Memb. Sci. 320 (2008) 292-302.

[18] E.R. Cornelissen, D. Harmsen, K.F. de Korte, C.J. Ruiken, J.-J. Qin, H. Oo, et al., Membrane fouling and process performance of forward osmosis membranes on activated sludge, J. Memb. Sci. 319 (2008) 158-168.

[19] R. Valladares Linares, V. Yangali-Quintanilla, Z. Li, G. Amy, Rejection of micropollutants by clean and fouled forward osmosis membrane, Water Res. 45 (2011) 6737-6744. 
[20] M. Qasim, N.A. Darwish, S. Sarp, N. Hilal, Water desalintion by forward (direct) osmosis phenomenon: a comprehensive review, Desalination 374 (2015) 47-69.

[21] N.Y. Yip, A. Tiraferri, W.A. Phillip, J.D. Schiffman, M. Elimelech, High performance thinfilm composite forward osmosis membrane, Environ. Sci. Technol. 44(2010) 3812-3818.

[22] J. Wei, C. Qiu, C.Y. Tang, R. Wang, A.G. Fane, Synthesis and characterization of flat-sheet thin film composite forward osmosis membranes. J. Membr. Sci. 372 (2011) 292-302.

[23] J. Benavente, M.I Vázquez, Effect of age and chemical treatments on characteristic parameters for active and porous sublayers of polymeric composite membranes, J. Colloid Interface Sci. 273 (2004) 547-555.

[24] X. Zhai, J. Meng, R. Li, L. Ni, Y. Zhang, Hypochlorite treatment on thin film composite RO membrane to improve boron removal performance, Desalination. 274 (2011) 136-143.

[25] Y.N. Kwon, J.O. Leckie, Hypochlorite degradation of crosslinked polyamide membranes. II. Changes in hydrogen bonding behavior and performance, J. Memb. Sci. 282 (2006) 456-464.

[26] V.T. Do, C.Y. Tang, M. Reinhard, J.O. Leckie, Degradation of polyamide nanofiltration and reverse osmosis membranes by hypochlorite., Environ. Sci. Technol. 46 (2012) 852-859.

[27] B. Jung, J.K. Yoon, B. Kim, H.W. Rhee, Effect of molecular weight of polymeric additives on formation, permeation properties and hypochlorite treatment of asymmetric polyacrylonitrile membranes, J. Memb. Sci. 243 (2004) 45-57.

[28] S. Rouaix, C. Causserand, P. Aimar, Experimental study of the effects of hypochlorite on polysulfone membrane properties, J. Membr. Sci. 277 (2006) 137-147.

[29] Y. Zhang, J. Wang, F. Gao, H. Tao, Y. Chen, H. Zhang, Impact of sodium hypochlorite ( $\mathrm{NaClO}$ ) on polysulfone (PSF) ultrafiltration membranes: The evolution of membrane performance and fouling behavior, Sep. Purif. Technol. 175 (2017) 238-247.

[30] B.-X. Cai, Permselectivity of polyamide composite membrane modified by solvent, J. Appl. Polym. Sci. 92 (2004) 1005-1010.

[31] K.L. Németh-Cahalan, K. Kalman, J.E. Hall, Molecular basis of pH and Ca2+ regulation of aquaporin water permeability., J. Gen. Physiol. 123 (2004) 573-580.

[32] C.X. Zhao, H.-B. Shao, L.Y. Chu, Aquaporin structure-function relationships: water flow through plant living cells., Colloids Surf. B. Biointerfaces. 62 (2008) 163-172. . 
[33] R.C. Ong, T.S. Chung, J.S. de Wit, B.J. Helmer, Novel cellulose ester substrates for high performance flat-sheet thin-film composite (TFC) forward osmosis (FO) membranes, J. Memb. Sci. 473 (2015) 63-71.

[34] Z. Wang, J, Tang, C. Zhu, Y. Dong, Q, Wang, Z, Wu, Chemical cleaning protocols for thin film composite (TFC) polyamide forward osmosis membranes used for municipal wastewater treatment, J. Membr. Sci. 475 (2015) 184-192.

[35] M.J. Borgnia, D. Kozono, G. Calamita, P.C. Maloney, P. Agre, Functional reconstitution and characterization of AqpZ, the E. coli water channel protein., J. Mol. Biol. 291 (1999) 1169-1179.

[36] X. Kong, Z. Li, D. Lu, Z. Liu, J. Wu, Multiscale simulation of surfactant-aquaporin complex formation and water permeability, RSC Adv. 4 (2014) 375-392.

[37] J.S. Hansen, A. Vararattanavech, I. Plasencia, P.J. Greisen, J. Bomholt, J. Torres, J. Emneus, C. Hélix-Nielsen, Interaction between sodium dodecyl sulfate and membrane reconstituted aquaporins: a comparative study of spinach SoPIP2;1 and E. coli AqpZ., Biochim. Biophys. Acta. 1808 (2011) 2600-2607.

[38] K. Ohki, F. Tokiwa, Physicochemical properties of alpha-olefi n-sulfonate sodium salts, J. Chem. Soc. Japan. (1970). https://scholar.google.com/scholar?hl=es\&q=Physicochemical+properties+of+++++alpha -olefin-sulfonate+sodium+salts\&btnG=\&Ir=\#0 (accessed June 8, 2015).

[39] D. Nanda, K.L. Tung, Y.L. Li, N.J. Lin, C.J. Chuang, Effect of pH on membrane morphology, fouling potential, and filtration performance of nanofiltration membrane for water softening, J. Memb. Sci. 349 (2010) 411-420.

[40] H. Zhu, M. Nyström, Cleaning results characterized by flux, streaming potential and FTIR measurements, Colloids Surfaces A Physicochem. Eng. Asp. 138 (1998) 309-321.

[41] M. Nyström, H. Zhu, Characterization of cleaning results using combined flux and streaming potential methods, J. Memb. Sci. 131 (1997) 195-205.

[42] M.H. Oo, S.L. Ong, Implication of zeta potential at different salinities on boron removal by RO membranes, J. Memb. Sci. 352 (2010) 1-6.

[43] Y.-N. Kwon, J.O. Leckie, Hypochlorite degradation of crosslinked polyamide membranes: I. Changes in chemical/morphological properties, J. Memb. Sci. 283 (2006) 21-26.

[44] N.P. Soice, A.C. Maladono, D.Y. Takigawa, A.D. Norman, W.B. Krantz, A.R. Greenberg, Oxidative degradation of polyamide reverse osmosis membranes: Studies of molecular model compounds and selected membranes, J. Appl. Polym. Sci. 90 (2003) 1173-1184. 
[45] R. Valladares Linares, Z. Li, M. Abu-Ghdaib, C.H. Wei, G. Amy, J.S. Vrouwenvelder, Water harvesting from municipal wastewater via osmotic gradient: An evaluation of process performance, J. Memb. Sci. 447 (2013) 50-56.

[46] V. Yangali-Quintanilla, Z. Li, R. Valladares, Q. Li, G. Amy, Indirect desalination of Red Sea water with forward osmosis and low pressure reverse osmosis for water reuse, Desalination. 280 (2011) 160-166. 